Stephen D. Theriault

\title{
Erratum to: A new proof of the odd primary homotopy exponent of spheres
}

Published online: 10 July 2013

\section{Erratum to: manuscripta math. 139, 137-151 (2012) \\ DOI 10.1007/s00229-011-0507-0}

I am grateful to Bill Richter for pointing out an error in [3], which was later used in [4] as one step of many in a new proof of the odd primary homotopy exponents of spheres.

The statement in question is as follows. Let $p$ be a prime and localize spaces and maps at $p$. Let $E^{2}: S^{2 n-1} \longrightarrow \Omega^{2} S^{2 n+1}$ be the double suspension, and let $W_{n}$ be its homotopy fibre. Gray [1] constructed a classifying space $B W_{n}$ for $W_{n}$, a homotopy fibration $S^{2 n-1} \stackrel{E^{2}}{\longrightarrow} \Omega^{2} S^{2 n+1} \longrightarrow B W_{n}$ and a homotopy fibration $B W_{n} \longrightarrow \Omega^{2} S^{2 n p+1} \stackrel{\phi}{\longrightarrow} S^{2 n p-1}$. Gray went on to conjecture that the composite $\Omega^{2} S^{2 n p+1} \stackrel{\phi}{\longrightarrow} S^{2 n p-1} \stackrel{E^{2}}{\longrightarrow} \Omega^{2} S^{2 n p+1}$ is homotopic to the $p$ th-power map.

In [3, Theorem 1.1] a proof was given that Gray's conjecture holds for primes $p \geq 3$. However, this made critical use of [3, Lemma 2.3], which Gray and Richter later identified as being incorrect without additional assumptions. It may be possible that these additional assumptions can be shown to hold for the specific application of [3, Lemma 2.3] used in the argument of [3, Theorem 1.1], but this is unclear. Instead, Richter [2] uses different methods to give a proof of Gray's conjecture which holds for all primes $p$.

In terms of [4], the statement that the composite $\Omega^{2} S^{2 n p+1} \stackrel{\phi}{\longrightarrow} S^{2 n p-1} \stackrel{E^{2}}{\longrightarrow}$ $\Omega^{2} S^{2 n+1}$ is homotopic to the $p$ th-power map for $p$ odd is used to prove [4, Lemma 6.3]. So if the citation to [3] for a proof of this statement is changed to a citation to [2], the argument in [4] goes through without change.

The Online version of the original article can be found under doi:10.1007/S00229-011-0507-0

S. D. Theriault: School of Mathematics, University of Southampton, Southampton SO17 1BJ, UK. e-mail: S.D.Theriault@ soton.ac.uk 


\section{References}

[1] Gray, B.: On the iterated suspension. Topology 27, 301-310 (1988)

[2] Richter, W.: A conjecture of Gray and the $p$ th-power map on $\Omega^{2} S^{2 n p+1}$, to appear in Proc. Am. Math. Soc.

[3] Theriault, S.D.: Proofs of two conjectures of Gray involving the double suspension. Proc. Am. Math. Soc. 131, 2953-2962 (2003)

[4] Theriault, S.D.: A new proof of the odd primary homotopy exponent of spheres. Manuscr. Math. 139, 137-151 (2012) 